\title{
Diffraction by an axicon with taking into consideration multiple internal reflections
}

\author{
Degtyarev S.A., Ustinov A.V., Khonina S.N. \\ Samara State Aerospace University, \\ Image Processing Systems Institute, Russian Academy of Sciences
}

\begin{abstract}
In this work we consider laser beam diffraction by narrow elongated axicon with conical angle, which is small enough for multiple internal reflection arising. Those sorts of tapers are widely used in micro and nanooptics. We have to take into account more than one internal reflection for correct description of beam propagation through the axicon. The diffraction is simulated with two approaches: pure "geometrical optics" and Helmholtz equation solving with "Finite Elements Method". Based on ray optics we derive analytic formulas for conical angles meanings, which provide maximums and minimums of intensity on optical axis. Derived numerical simulation verifies theoretically obtained results.
\end{abstract}

Keywords: tapers, conical angle, geometrical optics, internal reflection, Finite Elements Method

Citation: Degtyarev SA, Ustinov AV, Khonina SN. Diffraction by an axicon with taking into consideration multiple internal reflections. Proceedings of Information Technology and Nanotechnology (ITNT-2015), CEUR Workshop Proceedings, 2015; 1490: 27-36. DOI: 10.18287/1613-0073-2015-1490-27-36

\section{Introduction}

Axicons [1, 2] can be used to create nondiffraction Bessel beams in classical Optics [3, 4]. Metalized axicons with small cone angles are also utilised as near-field probes and tapers for sharp focusing in Nanophotonics $[5,6]$ and especially in nearfield microscopy [7-9] and spectroscopy [10]. To determine the optimum parameters of such tapers we need to provide simulation in accordance with the assumptions of the rigorous vectorial diffraction theory [11-14].

Working of metal conical structure is explained by the lightning-rod effect [15] which can be observed near dielectric structures [16]. The lightning-rod effect lies in the fact that longitudinal electric field component are enhanced near sharp structures. It can be observed, for example, in cases where radially polarized beams [17-19] or linearly polarized beams [20-22] are focused.

The dielectric characteristics of axicons can be approximately estimated with ray and scalar optical theory [23, 24], and then defined more accurately with more rigorous methods. 
In this work the ray optics approach was used as a rough calculation method. Using ray optics we derived analytic formulas for beam diffraction by the axicon with high numerical aperture (NA) including NAs that were over than the limiting value. Overcoming the limiting value of NA in ray optics means the axicon angle is small enough for total internal reflection arising. Consequently, we have to take into consideration more than one total internal reflection with further decrease of axicon's angle. We obtained axicon's angles which provide maximum and minimum values of intensity on the optical axis. Finite element method of solving Helmholtz equation was used to provide a more rigorous approach for verification of results of the analytical estimations.

\section{Theoretical analysis with ray optics approach}

Ray paths are described in detail in works [23]. But authors are not have deal with the case that axicon angle is much less than limited value.

Let us quote few results from [23] without any proofs. Notice that every ray impinges to the plain base of the axicon (left side in figure 1). $\alpha_{0}$ is a half angle of axicon's top. We call $\alpha_{0}$ axicon's angle. If $\alpha_{0}$ is large enough, total internal reflection is absent. In addition, rays pass through the right side and converge into the focal straight line segment.

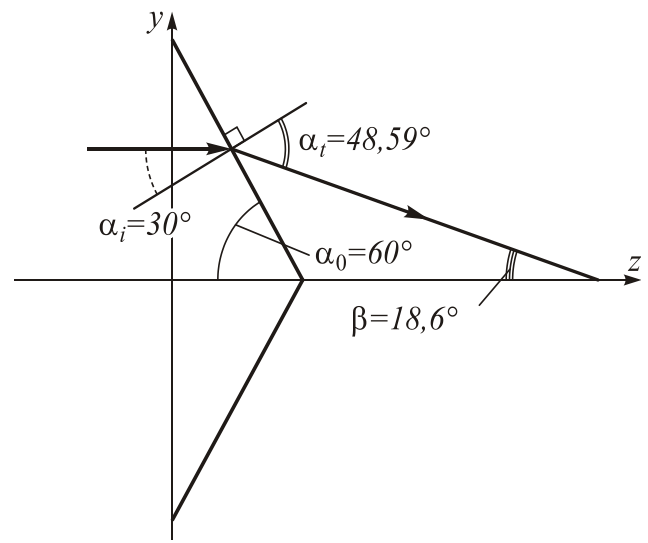

Fig. 1. - Rays paths in axicon in case of absence of total internal reflection

Such configuration remains as long as axicons angle satisfies inequality:

$\alpha_{0} \geq \arccos (1 / n)$,

Where $n$ is refractive index of the axicon's material.

If refractive index of $n=1.5$ the maximum angle is $48.19^{\circ}$. If the angle is less, total internal reflection occurs at the upside of the axicon (see figure 2). In this diagram it is apparent that the angle $\alpha_{0}$ is greater than

$\alpha_{0}>30^{\circ}+\frac{1}{3} \arcsin \frac{1}{n}$ 
therefore, total internal reflection takes place at bottom side of the axicon. Consequently, the rays turn back and leave the axicon and travel back to the left side.

If $n=1.5$ we can derive from Eq. (2) that $\alpha_{0}>43.94^{\circ}$. Ray paths for different angles $\alpha_{0}=44^{\circ}, 45^{\circ}, 47^{\circ}$ are also shown in figure 2 and it is obvious from the symmetry that rays travel back if $\alpha_{0}=45^{\circ}$.
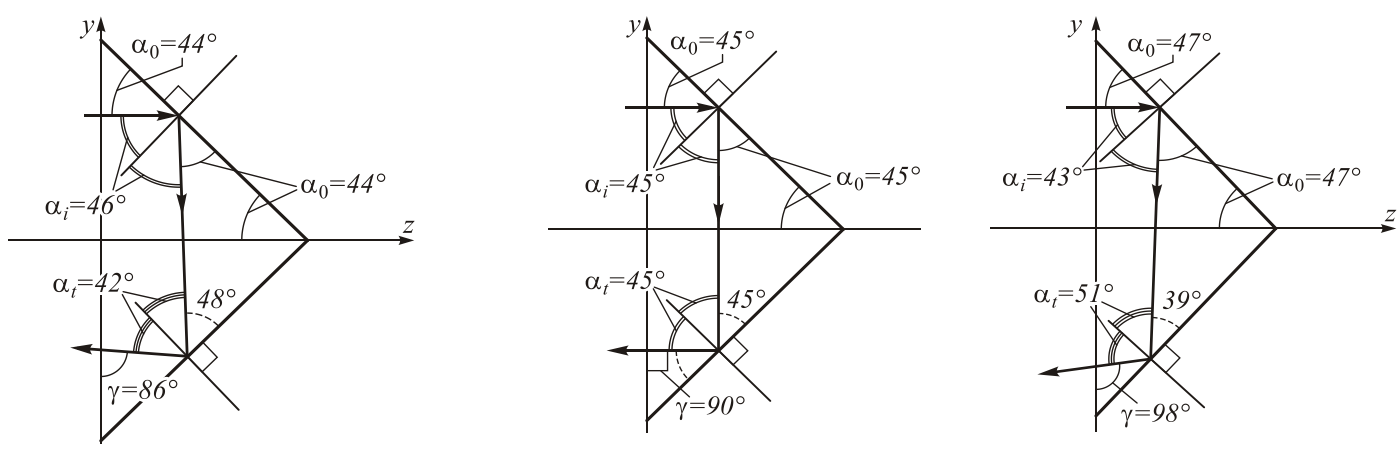

Fig. 2. - Rays paths in axicon in case of double total internal reflections from top and bottom sides $\alpha_{0}=44^{\circ}, 45^{\circ}, 47^{\circ}$

If the angle is less than this expression (2), full internal reflection from the bottom side does not occur; therefore rays leave the axicon through the bottom side and travel down. Nevertheless, if the angle is not much less than (2), the ray travels to the bottom-left direction. Thus, we can call this axicon opaque (figure 3 ).

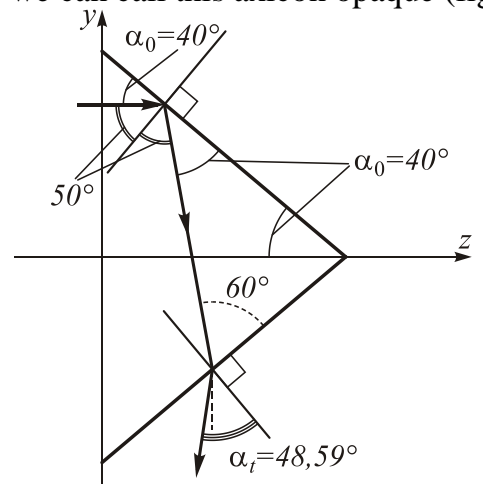

Fig. 3. - Rays paths in axicon in case of $\alpha_{0}=40^{\circ}$

In figure 3, the horizontal projection of the ray is decreasing with a decrease of the angle. Therefore, if the angle $\alpha_{0}=\alpha_{\text {ver }}$ horizontal projection becomes zero, hence the ray travels vertically down (figure 4 ). This angle is defined from the equation: $\sin \alpha_{v e r}+n\left(4 \cos ^{3} \alpha_{v e r}-3 \cos \alpha_{v e r}\right)=0$. 
If $n=1.5, \alpha_{\mathrm{ver}} \approx 38^{\circ}$.

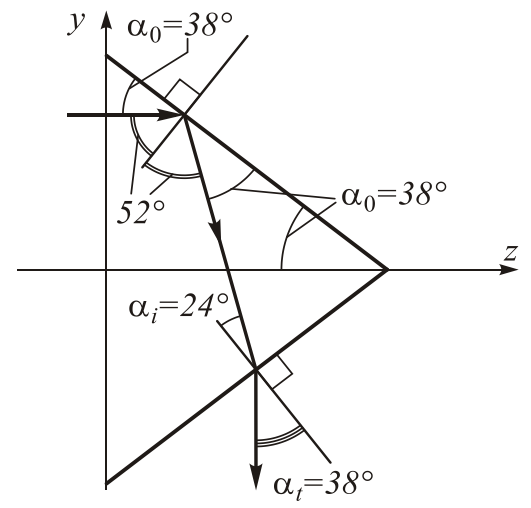

Fig. 4. - Rays paths in axicon in case of $\alpha_{0}=\alpha_{\text {ver }}$
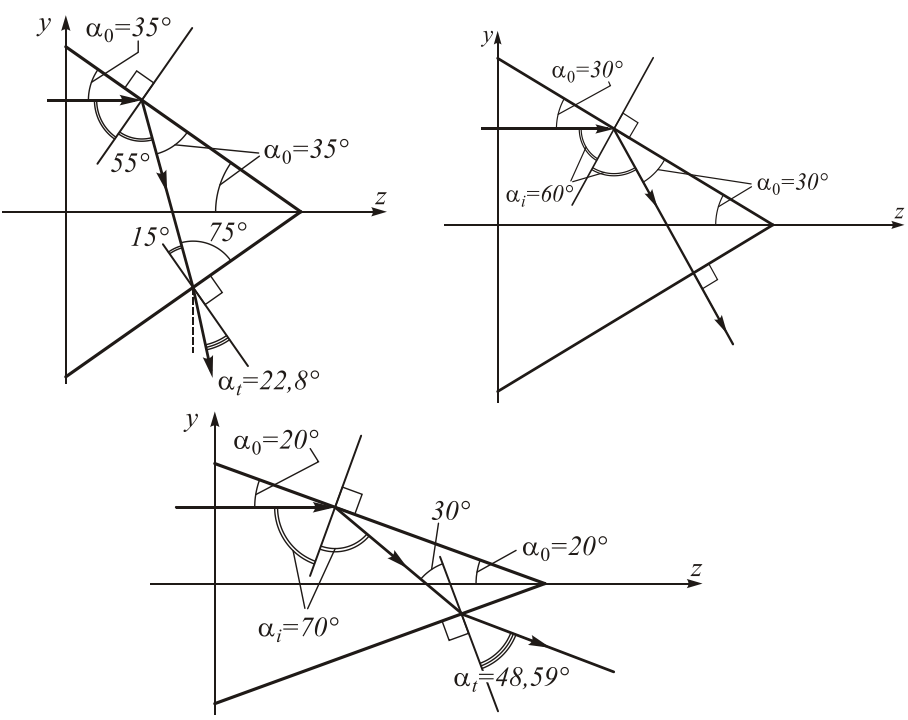

Fig. 5. - Rays paths in axicon in case of $\alpha_{0}=35^{\circ} ; 30^{\circ} ; 20^{\circ}$

For smaller meanings of the angle $\alpha_{0}$ horizontal projection of leaving ray is directed to the right. Although, rays do not intersect optical axis, thus focus is apparent (figure 5).

In [23] the authors consider only those configurations of axicon which are described above. However, it is noticed that by decreasing the axicon's angle rightdirected horizontal projection of leaving ray are increasing. If $\alpha_{0}=30^{\circ}$ this presents a special case. The rays pass through the bottom side without any refraction because the ray is perpendicular to the side, which happens if the refractive index lies in the range: 
$2 / \sqrt{3}<n<2$. If we continue decreasing $\alpha_{0}$, the vertical projection is also decreasing and once it reaches zero, the rays travel horizontally to the right side.

Let us have a look to the last scheme in figure 5. We can make sure that horizontally leaving ray can appears if following equation takes place: $\alpha_{0}+\alpha_{t}=90^{\circ}$ ( $\alpha_{t}$ is the refracting angle). If we take into account previous equation, refraction law and the fact that the angle of incidence to the bottom side is $90^{\circ}-3 \alpha_{0}$, if a single reflection is from the top side and $\alpha_{0}<30^{\circ}$, we can see that $\alpha_{0}$ has to satisfy following equation if the leaving ray is horizontally-oriented. $n \cos 3 \alpha_{0}=\cos \alpha_{0}$.

$$
\text { If } n=1.5, \alpha_{0}=16.78^{\circ} \text {. }
$$

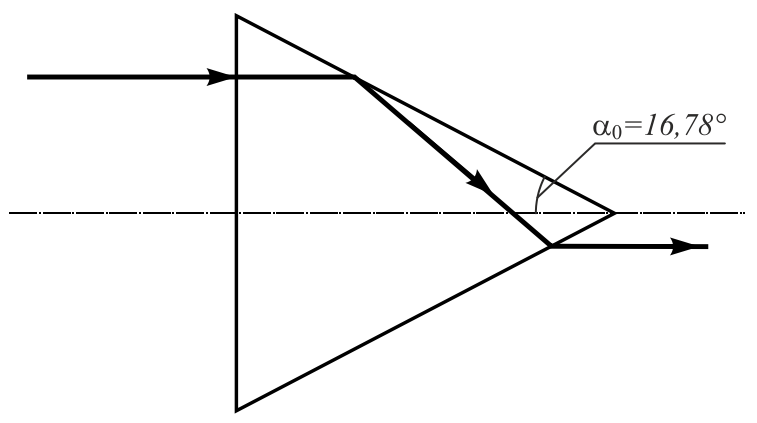

Fig. 6. - Horizontal ray path in axicon in case of $\alpha_{0}=16.78^{\circ}$

If the angle does not satisfy the equation (4), vertical projection of the leaving ray becomes directed upwards. Therefore, rays intersect with optical axis and form real focus (figure 7). In contrast to the results presented in the figure 1, in this situation leaving ray intersects the optical axis from the bottom to the top. Once more distinguishing feature is that the entrance pupil diameter is much greater than exit pupil diameter.

However, from the equation (4) we can determine that the angle's interval which provides real focuses of the axicon with single total internal reflection is very small. The condition for the second total internal reflection from the bottom side is therefore as follows:

$$
n \cos 3 \alpha_{0}=1 \Rightarrow \alpha_{0}=\frac{1}{3} \arccos \frac{1}{n}
$$




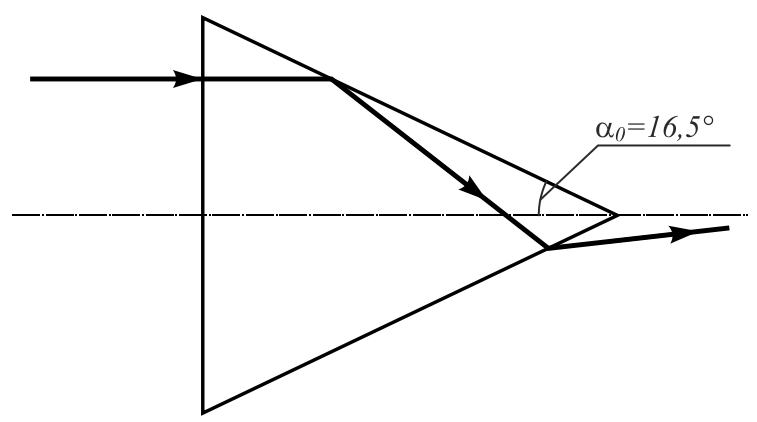

Fig. 7. - Real focus with single total internal reflection in the axicon

This is three times less than the angle which is satisfying inequality in (1). In this case $\alpha_{0}=16.06^{\circ}$, if $n=1.5$. Varying the angle from (4) to (5) we can achieve focal segment theoretically with any length. As we can see the angles interval which provides real axicon's focus, is very small and equals just 0.72 degree.

If the angle is smaller than (5), second full internal reflection occurs at the bottom side, the ray falls into the top side. Here ray is refracted and travels to the up-right direction. Thus, we have an apparent focus again. As it is shown in figure 6 , the leaving ray is oriented horizontally if the same equation $\alpha_{0}+\alpha_{t}=90^{\circ}$ is satisfied. But where the angle of incidence equals $90^{\circ}-5 \alpha_{0}$ at the top side in case of double total internal reflection from the top and then the bottom sides. Hence, the leaving ray will be oriented horizontally (figure 8 ) if the angle satisfies the equation: $\mathrm{n} \cos 5 \alpha_{0}=\cos \alpha_{0}$.

If $n=1.5$ this angle equals $9.785^{\circ}$.

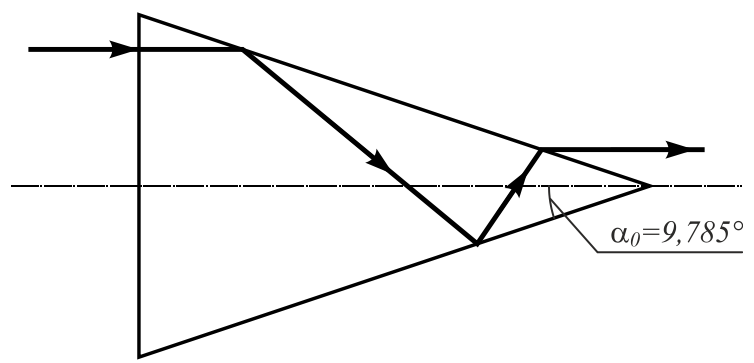

Fig. 8. - Horizontal ray path in axicon in case of $\alpha_{0}=9.785^{\circ}$

If the angle even smaller than is defined from equation (6), the vertical projection of the ray becomes oriented downward. Rays intersect the optical axis and form the real focus as indicated in figure 9. The leaving ray intersects the optical axis from up to down (as in figure 1), but only after two total internal refractions. 


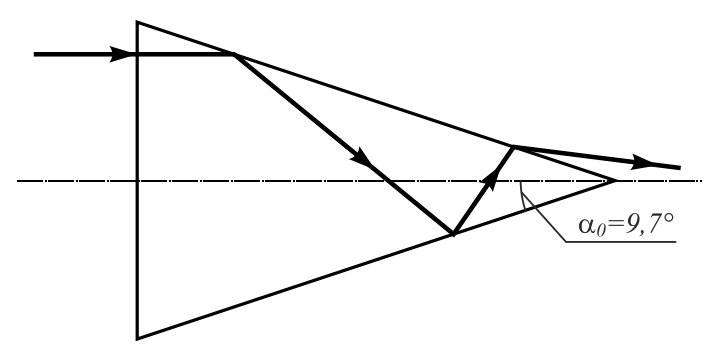

Fig. 9. - Real focus with double total internal reflection in the axicon

In the same way as in figure 7 we can therefore surmise derive that another total internal reflection occurs if following equation is satisfied:

$$
n \cos 5 \alpha_{0}=1 \Rightarrow \alpha_{0}=\frac{1}{5} \arccos \frac{1}{n} \text {. }
$$

That is five time less than it is derived from (1). If $n=1.5, \alpha_{0}=9.64^{\circ}$. Thus, angle interval which provides real focus with two total internal reflections is much smaller and equals 0.145 degree.

It is also possible to make sure that with the continuing decreasing of the angle we have infinite exchanging of real and apparent focuses. Boundaries of intervals of real and apparent focuses were defined by situations of total internal reflections (as in (5) and (7)) and the situations of leaving ray horizontality (as in (4) and (6)).

Real focus will be observed in narrow ranges of axicon's angles which are defined by the following inequalities:

$\alpha_{\min }^{\text {real }}<\alpha_{0}<\alpha_{\max }^{\text {real }}$,

here $\alpha_{\min }^{\text {real }}$ is achieved from equation:

$n \cos \left[(2 p+1) \alpha_{\min }^{\text {real }}\right]=\cos \left(\alpha_{\text {min }}^{\text {real }}\right)$,

and $\alpha_{\max }^{\text {real }}$ from the equation:

$n \cos \left[(2 p+1) \alpha_{\max }^{\text {real }}\right]=1$.

Equations (8a) and (8b) give boundaries for $p$-fold total internal reflection, $p \geq 1$. If $\mathrm{p}$ is even, the ray intersects the optical axis from up to down as it is shown in figure 9. If $\mathrm{p}$ is odd, the ray intersects the optical axis from down to up as in figure 7.

Apparent focus is observed with $p$-fold total internal reflection in larger ranges, which are further defined by adding the following inequalities:

$\alpha_{\min }^{a p p}<\alpha_{0}<\alpha_{\max }^{a p p}$.

here

$$
\begin{aligned}
& n \cos \left[(2 p-1) \alpha_{\min }^{a p p}\right]=1, \quad p \geq 2, \\
& n \cos \left[(2 p+1) \alpha_{\max }^{a p p}\right]=\cos \left(\alpha_{\max }^{a p p}\right), \quad p \geq 1
\end{aligned}
$$

The bottom boundary for single total internal reflection is not defined by formula (9a). It is equals the $\alpha_{\text {ver }}$, which is derived from (3). From this it is evident that the range of the intervals is becoming increasingly smaller. That has explicitly resulted from the meaning of the boundary of new total internal reflection, which can be easily 
obtained analytically: $\frac{1}{2 p+1} \arccos \frac{1}{n}$. This indicates the boundary is decreasing if $p$ is increasing. Thus, the received width of the 'real focus gap' is the largest and equals 0.72 degree for $p=1$. As noted, other gaps are becoming increasingly narrower.

Because of narrowness of angle ranges for real focuses, we should discuss the resonant phenomena. The same problems with polychromatic light can occur, because of dispersion transmission ranges can be different for different wavelengths. Furthermore, for one part of the spectrum the axicon can provide real focus and for another part of spectrum focus can be apparent. It is evident, therefore, that the axicon makes spectral redistribution as a prism.

Thus, from the finding we can see that ray optical approach has become useful for understanding of rays paths and intensity distribution in an axicon. However, it is problematic to receive total intensity distribution and estimations of beams sizes. For these reasons we have to apply a more rigorous theory.

\section{Numerical simulation by means of solving Helmholtz equation with finite element method}

In this work we provide numerical simulation of Gaussian beam diffraction by flat axicon with finite element method which is realized in Comsol.

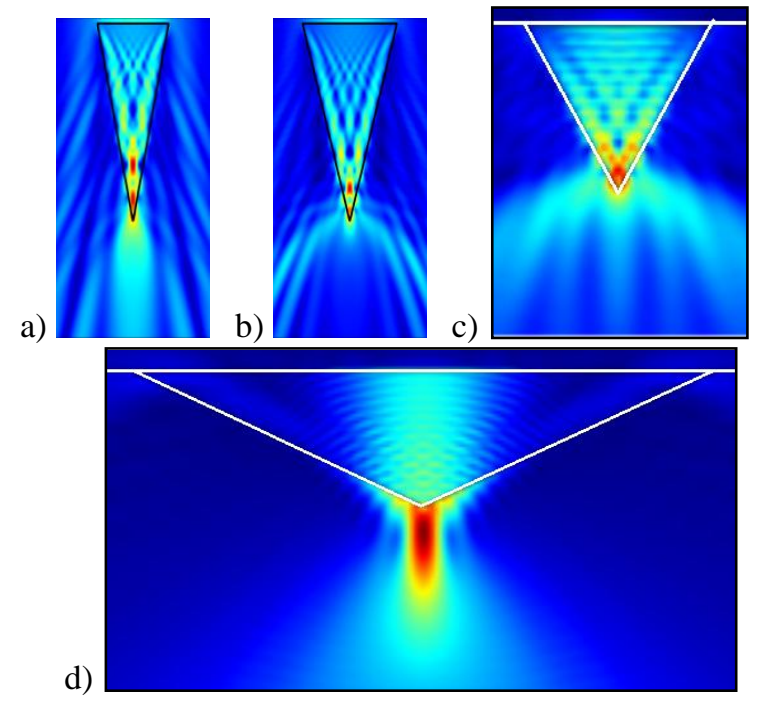

Fig. 10. - Simulation results of Gaussian beam diffraction by axicons with different cone angles: a) $\alpha_{0}=10.5^{\circ}$, b) $\alpha_{0}=11^{\circ}$, c) $\alpha_{0}=30^{\circ}$, d) $\alpha_{0}=61^{\circ}$

In the figure 10 we show the simulation results of TE-polarized Gaussian beam diffraction by $2 \mathrm{D}$ axicons with different axicon's angle $\alpha_{0}$. Wavelength is $\lambda=532$ $\mathrm{nm}$, and refraction index is $n=1.5$.

As we can see in figure 10, whereas we increase axicon angle from $10.5^{\circ}$ to $11^{\circ}$, the internal reflection arises and the main part of energy scatters at a high angle to the 
optical axis. Ray optics predicts that this beam splitting is in contrast to wave optics which takes into evanescent field. An axicon works as a refractive element and produces a strong light segment where the axicon angle is more $49^{\circ}$.

\section{Conclusions}

In this work we considered diffraction of Gaussian beams by the refractive axicon in case of numerical aperture of the axicon is much greater than limited value which corresponds to total internal reflection. This situation did not take into account because it was reckoned that axicon did not transmit light in case of out-the-limited.

Diffraction was considered using two approaches: ray optics and numerical solving of the Helmholtz equation with finite elements method. In the first case analytical estimations are derived for high numerical aperture including the case of out-of-limited numerical aperture.

However, out-of-limited numerical aperture in ray optics corresponds to total internal reflection arising, careful analysis of ray paths reveals that with increasing numerical aperture (decreasing axicon's angle) part of incident energy passes through the axicon though and the transmitted rays form either real or apparent focus.

Finally, the rigorous wave approach allowed us to verify the perceived analytical results.

\section{Acknowledgements}

This work was supported by Russian Science Foundation grant No. 14-19-00114.

\section{References}

1. McLeod JH. The axicon: a new type of optical element. Journal of the Optical Society of America, 1954; 44: 592-597.

2. Jaroszewicz Z, Burvall A, Friberg AT. Axicon - the most important optical element. Optics \& Photonics News, 2005; April.

3. Durnin J, Miceli JJ, Eberly JH. Diffraction-free beams. Physical Review Letters, 1987; 58: 1499-1501.

4. McGloin D, Dholakia K. Bessel beams: diffraction in a new light. Contemporary Physics, 2005; 46(1): 15-28.

5. Babadjanyan AJ, Margaryan NL, Nerkararyana KhV. Superfocusing of surface polaritons in the conical structure. Journal Applied Physics, 2000; 87(8): 3785-3788.

6. Novotny L, Hecht D. Principles of Nano-Optics. Cambridge: New York, 2006.

7. Goncharenko AV, Chang H-C, Wang J-K. Electric near-field enhancing properties of a finite-size metal conical nano-tip. Ultramicroscopy, 2007; 107: 151-157.

8. Antosiewicz TJ, Wróbel P, Szoplik T. Nanofocusing of radially polarized light with dielectric-metal-dielectric probe. Optics Express, 2009; 17(11): 9191-9196.

9. Choo H, Kim M-K, Staffaroni M, Seok TJ, Bokor J, Cabrini S, Schuck PJ, Wu MC, Yablonovitch E. Nanofocusing in a metal-insulator-metal gap plasmon waveguide with a three-dimensional linear taper. Nature Photonics, 2012; 6: 838-844.

10. Berweger S, Atkin JM, Olmon RL, Raschke MB. Light on the tip of a needle: plasmonic nanofocusing for spectroscopy on the nanoscale. The Journal of Physical Chemistry Letters, 2012; 3: 945-952. 
11. Gramotnev DK, Vogel MW, Stockman MI. Optimized nonadiabatic nanofocusing of plasmons by tapered metal rods. Journal Applied Physics, 2008, 104: 034311-034318.

12. Davoyan AR, Shadrivov IV, Kivshar YS. Gramotnev DK. Optimal tapers for compensating losses in plasmonic waveguides. Physica Status Solidi - Rapid Research Letters, 2010; 4(10): 277- 279.

13. Degtyarev SA, Khonina SN, Alferov SV, Karpeev SV. Theoretical and experimental study of aperture size effects on the polarization sensitivity of near-field microscopy fiberoptic probes. Proceedings of SPIE, 2014; 9156: 915608; doi:10.1117/12.2054204.

14. Degtyarev SA, Khonina SN. Transmission of focused light signal through an apertured probe of a near-field scanning microscope. Pattern Recognition and Image Analysis, 2015; 25(2): 306-313.

15. Gramotnev DK, Bozhevolnyi SI. Nanofocusing of electromagnetic radiation. Nature Photonics, 2014; 8: 14-23.

16. Degtyarev SA, Khonina SN, Ustinov AV, Kazanskiy NL. Lightning-rod effect near sharp dielectric structures. Proceedings of SPIE, 2015; 9533: 95330A; doi: $10.1117 / 12.2180353$

17. Zhang Y, Wang L, Zheng C. Vector propagation of radially polarized Gaussian beams diffracted by an axicon. Journal of the Optical Society of America A, 2005; 22(11): 25422546.

18. Kotlyar VV, Stafeev SS. Modeling sharp focusing of a radially polarized laser mode with a conic and binary microaxicons. Computer Optics, 2009; 33(1): 52-60.

19. Khonina SN, Degtyarev SA. A longitudinally polarized beam generated by a binary axicon. Journal of Russian Laser Research, 2015; 36(2): 151-161.

20. Khonina SN, Nesterenko DV, Morozov AA, Skidanov RV, Soifer VA. Narrowing of a light spot at diffraction of linearly-polarized beam on binary asymmetric axicons. Optical Memory and Neural Networks (Information Optics), 2012; 21(1): 17-26.

21. Khonina SN, Karpeev SV, Alferov SV, Savelyev DA, Laukkanen J, Turunen J. Experimental demonstration of the generation of the longitudinal E-field component on the optical axis with high-numerical-aperture binary axicons illuminated by linearly and circularly polarized beams. Journal of Optics, 2013; 15: 085704-085712.

22. Khonina SN, Savelyev DA. High-aperture binary axicons for the formation of the longitudinal electric field component on the optical axis for linear and circular polarizations of the illuminating beam. Journal of Experimental and Theoretical Physics, 2013; 117(4): 623-630.

23. Ustinov AV, Khonina SN. Calculating the complex transmission function of refractive axicons. Optical Memory and Neural Networks (Information Optics), 2012, 21(3): 133144.

24. Ustinov AV, Khonina SN. Analysis of laser beam diffraction by axicon with the numerical aperture above limiting. Computer Optics, 2014; 38(2): 213-222. 